\title{
ON BERNSTEIN TYPE THEOREMS CONCERNING THE GROWTH OF DERIVATIVES OF ENTIRE FUNCTIONS
}

\author{
SEN-ZHONG HUANG
}

(Communicated by Theodore W. Gamelin)

\begin{abstract}
A subspace $X$ of $L_{l o c}^{1}(\mathbb{R})$ which is invariant under all left translation operators $T_{t}, t \in \mathbb{R}$, is called admissible if $X$ is a Banach space satisfying the following properties:

(i) If $\left\|f_{n}\right\|_{X} \rightarrow 0$, then there exists a subsequence $\left(n_{k}\right)$ such that $f_{n_{k}}(s) \rightarrow 0$ almost everywhere.

(ii) The group $\mathcal{T}_{X}:=\left\{\left.T_{t}\right|_{X}: t \in \mathbb{R}\right\}$ is a bounded strongly continuous group. In this case, let

$$
C_{X}:=\sup \left\{\left\|T_{t}\right\|_{X}: t \in \mathbb{R}\right\} .
$$

Typical admissible spaces are $C_{0}(\mathbb{R}), B U C(\mathbb{R})$ and all spaces $L^{p}(\mathbb{R})$ for $1 \leq p<\infty$. More generally, all of the Peetre interpolation spaces of two admissible spaces $X_{1}, X_{2}$ are also admissible.

A function $g \in L_{l o c}^{1}(\mathbb{R})$ is called subexponential if for every $\delta>0, e^{-\delta|t|} g(t)$ $\in L^{1}(\mathbb{R})$. With these definitions our main result goes as follows:
\end{abstract}

Theorem 1. If $g$ is an entire function of exponential type $\tau$ such that its restriction to the real axis, denoted by $g_{\mathbb{R}}$, is subexponential and belongs to some admissible space $X$, then the derivative $g_{\mathbb{R}}^{\prime}$ is also in $X$. Moreover, $\left\|\alpha g_{\mathbb{R}}+g_{\mathbb{R}}^{\prime}\right\|_{X} \leq\left(\alpha^{2}+\tau^{2}\right)^{1 / 2} \cdot C_{X} \cdot\left\|g_{\mathbb{R}}\right\|_{X}$ for each real $\alpha$.

This result yields as consequences and in a systematic way many new and old Bernstein type inequalities.

\section{The MAIN THEOREMS}

Recall that an entire function $f$ is called to be of exponential type $\tau$ if

$$
\limsup _{r \rightarrow \infty} \frac{\log M(r)}{r}=\tau
$$

where $M(r):=\max \{|f(z)|:|z| \leq r\}$ is the maximum modulus of $f$. The classical Bernstein theorem [Bo, p.206, Theorem 11.1.2] states that if $f$ is an entire function of exponential type $\tau$ and is bounded on the real axis by $M$, then $\left|f^{\prime}(t)\right| \leq \tau M$ for all $t \in \mathbb{R}$, or equivalently, $\left\|f^{\prime}\right\|_{\infty} \leq \tau\|f\|_{\infty}$ for each entire function $f$ of exponential type $\tau$. The latter is referred as to Bernstein's inequality. Many extensions are given, see [Bo, Chap. 11], [D-S], [Le, Chap. IV] and [K-S-T]. It is the purpose of

Received by the editors August 16, 1995.

1991 Mathematics Subject Classification. Primary 30D20; Secondary 47D03, 47A10.

Key words and phrases. Entire function, Bernstein's inequality, strongly continuous group, spectrum.

Supported by a fellowship of the Deutscher Akademisher Austauschdienst (DAAD). 
this paper to give a systematical treat of these results and to find more Bernstein type inequalities.

We refer to Timan's book [Ti] or Lorentz's book [Lor] for applications of Bernstein's inequality to the Theory of Approximation of Functions.

As a preparation, recall from [Do, p.104] that an element $h$ in a unital Banach algebra $\mathcal{A}$ is called hermitian if the group $\left\{e^{i t h}: t \in \mathbb{R}\right\}$ is isometric, i.e.,

$$
\left\|e^{i t h}\right\|=1 \quad \text { for all } t \in \mathbb{R} \text {. }
$$

Many equivalent conditions are known, see [Do, p.104, Theorem 4.7] or [Pal, pp.267268]. For example, it is shown that $h$ is hermitian if and only if the numerical range

$$
W(h):=\left\{\omega(h): \omega \in \mathcal{A}_{W}^{*}\right\}
$$

is contained in the real axis, where the set $\mathcal{A}_{W}^{*}$ consists of all $\omega \in \mathcal{A}^{*}$ such that $\omega(1)=\|\omega\|=1$. The remarkable fact about hermitian elements is the following formula of Sinclair [Si]: For each hermitian element $h \in \mathcal{A}$ and each real $\alpha \in \mathbb{R}$ there holds

$$
\|i \alpha+h\|=r(i \alpha+h):=\sup \left\{\sqrt{\alpha^{2}+\lambda^{2}}: \lambda \in \sigma(h)\right\} .
$$

The proof of this formula for the case $\alpha=0$, due to Bonsall and Crabb [B-C] (see also [Do, pp. 105-106] or [Pal, pp.265-267]), is elementary. However, Sinclair's original proof is based on the following result of Duffin and Schaeffer [D-S, Theorem 1], which extends the above Bernstein theorem and asserts that

$$
\left|f^{\prime}(t)+\alpha f(t)\right| \leq\left(\alpha^{2}+\tau^{2}\right)^{1 / 2} \cdot \sup \{|f(s)|: s \in \mathbb{R}\}, \quad \alpha, t \in \mathbb{R},
$$

for every entire function $f$ of exponential type $\tau$. In [Re], there is a very simple proof of Duffin and Schaeffer's theorem. At this point the author thanks the referee for drawing his attention to [Re].

To continue, let $L_{l o c}^{1}(\mathbb{R})$ be the complex linear space of all locally Lebesgue integrable functions on $\mathbb{R}$. For each $t \in \mathbb{R}$ and $f \in L_{\text {loc }}^{1}(\mathbb{R})$ define $f^{t}$ to be the left translation of $f$, i.e.,

$$
f^{t}(s):=f(s+t) \quad \text { for all } s \in \mathbb{R} .
$$

Define the left translation operators through

$$
T_{t} f:=f^{t} \quad \text { for all } f \in L_{l o c}^{1}(\mathbb{R}), t \in \mathbb{R} .
$$

The set of operators $\mathcal{T}:=\left\{T_{t}: t \in \mathbb{R}\right\}$ satisfies the group property

$$
T_{s} T_{t}=T_{s+t} \quad \text { for all } s, t \in \mathbb{R} .
$$

Definition 1. A translation-invariant linear subspace $X$ of $L_{l o c}^{1}(\mathbb{R})$ is called admissible if $X$ is a Banach space satisfying the following properties:

(i) If $\left\|f_{n}\right\|_{X} \rightarrow 0$, then there exists a subsequence $\left(n_{k}\right)$ such that $f_{n_{k}}(s) \rightarrow 0$ almost everywhere.

(ii) The group $\mathcal{T}_{X}:=\left\{\left.T_{t}\right|_{X}: t \in \mathbb{R}\right\}$ is a bounded strongly continuous group on $X$. In this case, let

$$
C_{X}:=\sup \left\{\left\|T_{t}\right\|_{X}: t \in \mathbb{R}\right\}
$$


Typical examples of admissible spaces are $C_{0}(\mathbb{R})$, the space $B U C(\mathbb{R})$ of all bounded and uniformly continuous functions with the supremum norm and the $L^{p}$-spaces $L^{p}(\mathbb{R})$ for $1 \leq p<+\infty$. On each of these spaces $\mathcal{T}$ defines an isometric strongly continuous group. More examples can be obtained by taking $\mathcal{T}$-invariant subspaces of the above typical examples or through interpolation of these spaces. We note that many Orlicz spaces and Lorentz spaces are also admissible. In the subsequent section we will return to these constructions.

Next, we recall that the convolution of two functions $f, g \in L^{1}(\mathbb{R})$ is defined through

$$
f * g(t):=\int_{-\infty}^{\infty} f(s) g(t-s) d t, t \in \mathbb{R} .
$$

With this operation $L^{1}(\mathbb{R})$ becomes a commutative Banach algebra, called the group algebra on $\mathbb{R}$. The Fourier transform of a function $f \in L^{1}(\mathbb{R})$ is given by

$$
\hat{f}(s):=\int_{-\infty}^{\infty} e^{-i s t} f(t) d t, \quad s \in \mathbb{R} .
$$

If $\hat{f} \in L^{1}(\mathbb{R})$, then the inverse formula holds (see [Ka, Theorem 1.11, p.125]):

$$
f(t)=\frac{1}{2 \pi} \int_{-\infty}^{\infty} e^{i t s} \hat{f}(s) d s \quad \text { for almost every } \quad t \in \mathbb{R} .
$$

Let $X$ be an admissible space. For $f \in L^{1}(\mathbb{R})$, define the operator $T_{f} \in \mathcal{L}(X)$ by the following Bochner integral:

$$
T_{f}(x):=\int_{-\infty}^{\infty} T_{t}(x) f(t) d t \quad \text { for all } \quad x \in X .
$$

It is easily verified that the mapping $f \mapsto T_{f}$ is a continuous algebra homomorphism. Hence, the set

$$
I_{T}:=\left\{f \in L^{1}(\mathbb{R}): T_{f}=0\right\}
$$

is a closed ideal in $L^{1}(\mathbb{R})$. The Arveson spectrum $[\operatorname{Ar}]$ of $\mathcal{T}_{X}$ is defined as the hull of $I_{T}$, i.e.,

$$
\operatorname{Sp}\left(\mathcal{T}_{X}\right):=\operatorname{hull}\left(I_{T}\right)=\left\{t \in \mathbb{R}: \hat{f}(t)=0, \forall f \in I_{T}\right\} .
$$

For $x \in X$, consider

$$
I_{T}(x):=\left\{f \in L^{1}(\mathbb{R}): T_{f}(x)=0\right\},
$$

which is a closed ideal. The local spectrum of $\mathcal{T}_{X}$ at $x$ is defined as

$$
\operatorname{Sp}_{\mathcal{T}}(x):=\operatorname{hull}\left(I_{T}(x)\right)=\left\{t \in \mathbb{R}: \hat{f}(t)=0, \forall f \in I_{T}(x)\right\} .
$$

For a closed subset $\Lambda \subset \mathbb{R}$, the corresponding spectral subspace $X^{\mathcal{T}}(\Lambda)$ is

$$
X^{\mathcal{T}}(\Lambda):=\left\{x \in X: \operatorname{Sp}_{\mathcal{T}}(x) \subseteq \Lambda\right\} .
$$

Clearly, all spectral subspaces of an admissible space are also admissible. Moreover, it is known [Ped, Theorem 8.1.4-(iv), p.301] (see also [Hu, Prop. 1.2.6-(h)]) that the Arveson spectrum of $\mathcal{T}_{X}$ is the smallest closed subset $\Lambda \subset \mathbb{R}$ such that $X^{\mathcal{T}}(\Lambda)=X$. This implies that

$$
\operatorname{Sp}\left(\left.\mathcal{T}\right|_{X^{\mathcal{T}}(\Lambda)}\right) \subseteq \Lambda \quad \text { for all closed subsets } \Lambda \subseteq \mathbb{R} .
$$

A very useful criterion for an element $x \in X$ being in $X^{\mathcal{T}}(\Lambda)$ is that $T_{f}(x)=0$ for all $f \in j(\Lambda)$, where $j(\Lambda)$ is the ideal of all functions $f \in L^{1}(\mathbb{R})$ whose Fourier 
transform $\hat{f}$ is compact and disjoint from $\Lambda$. For the proof, see the remark following Definition 2.1 in $[\mathrm{Ar}]$ or [Hu, Proposition 1.2.6-(e)].

Let $A$ with the domain $D(A)$ be the infinitesimal generator of the strongly continuous group $\mathcal{T}_{X}$ (see $[\mathrm{Paz}]$ ). Let $g \in D(A)$. Then $g$ is a.e. differentiable and $A g=g^{\prime}$. Thus $A$ coincides with the derivative in $X$.

Lemma 1. Let $X$ be an admissible space and $A$ be the infinitesimal generator of $\mathcal{T}_{X}$. Then

$$
S p\left(\mathcal{T}_{X}\right)=i \sigma(A) .
$$

For $\tau \geq 0$, let $B$ be the infinitesimal generator of the group obtained by restricting $\mathcal{T}_{X}$ to the spectral subspace $X^{\mathcal{T}}([-\tau, \tau])$. Then $B$ is a bounded operator. Moreover, for $\alpha \in \mathbb{R}$,

$$
\|\alpha+B\|_{X} \leq\left(\alpha^{2}+\tau^{2}\right)^{1 / 2} \cdot C_{X},
$$

where $C_{X}$ is the constant in Definition 1.

Proof. The proof of the spectral identity $\operatorname{Sp}\left(\mathcal{T}_{X}\right)=i \sigma(A)$ can be found in [Ev] (see also [Hu, Proposition 1.3.9] and [Jo]). Moreover, a result of Olesen [Ol] (or see Corollary 3.3 in $[\mathrm{N}-\mathrm{H}]$ ) asserts that $A$ is bounded if and only if $\sigma(A)$ is bounded.

Consider the infinitesimal generator $B$. Combining $(* *)$ with the previous spectral identity we have

$$
\sigma(i B)=i \sigma(B)=\operatorname{Sp}\left(\left.\mathcal{T}\right|_{X^{\mathcal{T}}([-\tau, \tau])}\right) \subseteq[-\tau, \tau] .
$$

Therefore, $B$ is bounded. Note that each $T_{t}$ is an isometry under the new norm

$$
\left\||\|x\||=\sup \left\{\left\|T_{t} x\right\|: t \in \mathbb{R}\right\}, x \in X .\right.
$$

The operator $i B$ is hermitian with respect to this new norm. Thus, by Sinclair's formula $(*)$ we have

$$
\|i \alpha+i B\| \mid=r(i \alpha+i B) \leq\left(\alpha^{2}+\tau^{2}\right)^{1 / 2}
$$

for all $\alpha \in \mathbb{R}$. This implies the desired result.

We call a function $g \in L_{l o c}^{1}(\mathbb{R})$ subexponential if

$$
\int_{-\infty}^{\infty} e^{-\delta|t|}|g(t)| d t<+\infty \text { for all } \delta>0
$$

The Carleman transform of a subexponential function $g$ is defined as (see $[\mathrm{Hu}$, Chap. IV] and [Ka, p.179])

$$
\tilde{g}(\lambda):= \begin{cases}\int_{0}^{\infty} e^{i \lambda t} g(t) d t, & \operatorname{Im} \lambda>0 \\ -\int_{\infty}^{0} e^{i \lambda t} g(t) d t, & \operatorname{Im} \lambda<0 .\end{cases}
$$

Our main result goes as follows.

Theorem 1. Let $X$ be an admissible space and $g$ an entire function of exponential type $\tau \geq 0$. Assume that the restriction $g_{\mathbb{R}}$ of $g$ to the real axis is subexponential and belongs to $X$. Then, the derivative $g_{\mathbb{R}}^{\prime}$ is also in $X$ and

$$
\left\|\alpha g_{\mathbb{R}}+g_{\mathbb{R}}^{\prime}\right\|_{X} \leq\left(\alpha^{2}+\tau^{2}\right)^{1 / 2} \cdot C_{X} \cdot\left\|g_{\mathbb{R}}\right\|_{X}
$$

for all $\alpha \in \mathbb{R}$, where $C_{X}$ is the constant in Definition 1 . 
Proof. By Lemma 1 we need only check that the function $g_{\mathbb{R}}$ belongs to $X^{\mathcal{T}}([-\tau, \tau])$. This will be achieved in two steps.

Step 1. Let

$$
g(z)=\sum_{n=0}^{\infty} a_{n} \frac{z^{n}}{n !}, z \in \mathbb{C},
$$

be the series expansion of the entire function $g$ around the point $z=0$. For $n \geq 1$, we have by Cauchy's formula that

$$
\frac{a_{n}}{n !}=\frac{1}{2 \pi i} \int_{|z|=n / \tau} \frac{g(z)}{z^{n+1}} d z
$$

So,

$$
\left|a_{n}\right| \leq \frac{\tau^{n} \cdot n !}{n^{n}} M(n / \tau),
$$

where $M(\cdot)$ is the maximum modulus of $g$. Using Stirling's formula [Bo, p.6]

$$
n !=n^{n} e^{-n}(2 \pi n)^{1 / 2} e^{\delta_{n} /(12 n)}, 0<\delta_{n}<1,
$$

we have

$$
\limsup _{n \rightarrow \infty}\left|a_{n}\right|^{1 / n} \leq \frac{\tau}{e} \cdot \limsup _{n \rightarrow \infty} M(n / \tau)^{1 / n}=\tau .
$$

This implies that the convergence radius of the series $\sum_{n=0}^{\infty} a_{n} z^{n}$ is greater than or equal to $1 / \tau$. Thus, by computing the Carleman transform of $g_{\mathbb{R}}$ we obtain

$$
\tilde{g}(\lambda)=\sum_{n=0}^{\infty} a_{n}(-i \lambda)^{-n-1} \quad \text { for } \text { all } \quad \lambda \notin \mathbb{R} \quad \text { with } \quad|\lambda|>\tau .
$$

So, $\tilde{g}(\lambda)$ can be extended holomorphically to the region $|\lambda|>\tau$. By considering the left translations $g^{s}$ we find that all functions $\widetilde{g^{s}}(\lambda)$ can be extended holomorphically to the region $|\lambda|>\tau$.

Step 2. Let $f \in j([-\tau, \tau])$. Then, $\hat{f} \in L^{1}(\mathbb{R})$. By the inverse formula for the Fourier transform

$$
f(t)=\frac{1}{2 \pi} \int_{\mathbb{R}} \hat{f}(r) e^{i t r} d r \quad \text { a.e. }
$$

It follows that $f$ is equal a.e. to a continuous bounded function. Moreover, since $\operatorname{supp} \hat{f} \cap[-\tau, \tau]=\emptyset$, we can find $\tau<\tau_{1}<\tau_{2}$ such that

$$
\operatorname{supp} \hat{f} \subseteq\left[-\tau_{2},-\tau_{1}\right] \cup\left[\tau_{1}, \tau_{2}\right] .
$$

For $n \in \mathbb{N}$, let

$$
h_{n}:=\int_{-\infty}^{\infty} e^{-|t| / n} T_{t}(g) f(t) d t .
$$

Then,

$$
\lim _{n \rightarrow \infty}\left\|T_{f}(g)-h_{n}\right\|_{X}=0
$$


For fixed $n$ we obtain by the boundedness and the above representation of $f$ that

$$
\begin{aligned}
h_{n}(s) & =\int_{-\infty}^{\infty} f(t) e^{-|t| / n} g^{s}(t) d t \\
& =\int_{-\infty}^{\infty}\left[\frac{1}{2 \pi} \int_{-\infty}^{\infty} \hat{f}(r) e^{i r t} d r\right] e^{-|t| / n} g^{s}(t) d t \\
& =\frac{1}{2 \pi} \int_{-\infty}^{\infty} \hat{f}(r)\left[\int_{-\infty}^{\infty} e^{i r t-|t| / n} g^{s}(t) d t\right] d r \quad \text { (by Fubini's theorem) } \\
& =\frac{1}{2 \pi} \int_{-\infty}^{\infty} \hat{f}(r)\left[\widetilde{g^{s}}(r+i / n)-\widetilde{g}^{s}(r-i / n)\right] d r \\
& =\frac{1}{2 \pi}\left(\int_{-\tau_{2}}^{-\tau_{1}}+\int_{\tau_{1}}^{\tau_{2}}\right) \hat{f}(r)\left[\widetilde{g^{s}}(r+i / n)-\widetilde{g}^{s}(r-i / n)\right] d r .
\end{aligned}
$$

As seen in Step 1, the function $\widetilde{g}^{s}(\lambda)$ has a holomorphic extension to the region $|\lambda|>\tau$. This implies that the last term in the above equality goes to zero as $n \rightarrow \infty$. Hence,

$$
\lim _{n \rightarrow \infty} h_{n}(s)=0 \quad \text { for } \text { all } s \in \mathbb{R} .
$$

By Definition 1-(i) there exists a subsequence $\left(h_{n_{k}}\right)$ such that

$$
T_{f}(g)(s)=\lim _{k \rightarrow \infty} h_{n_{k}}(s)=0 \quad \text { a.e. }
$$

Consequently, $T_{f}(g)=0$ and thus

$$
T_{f}(g)=0 \text { for all } f \in j([-\tau, \tau]) .
$$

By the above mentioned criterion we see that $g_{\mathbb{R}} \in X^{\mathcal{T}}([-\tau, \tau])$. This finishes the proof.

The following consequence extends Theorem 1 and can be applied to derive some useful inequalities involving mean values, which we will give in the next section.

Theorem 2. Let $X$ be an admissible space with the constant $C_{X}$ and $g$ be an entire function of exponential type $\tau$ whose restriction $g_{\mathbb{R}}$ to the real axis belongs to $X$. Assume $N(\cdot)$ to be a translation-invariant continuous semi-norm on $X$; i.e., for some constant $D>0$ there holds:

$$
N\left(f^{t}\right)=N(f) \quad \text { and } \quad N(f) \leq D\|f\|_{X} \quad \text { for all } \quad f \in X, t \in \mathbb{R} .
$$

Then,

$$
N\left(\alpha g_{\mathbb{R}}+g_{\mathbb{R}}^{\prime}\right) \leq C_{X} \cdot\left(\alpha^{2}+\tau^{2}\right)^{1 / 2} N\left(g_{\mathbb{R}}\right) \quad \text { for all } \quad \alpha \in \mathbb{R} .
$$

Proof. For $r>0$ consider

$$
\|f\|_{r}:=r\|f\|_{X}+N(f), f \in X .
$$

By our assumptions on $N(\cdot),\|\cdot\|_{r}$ is a new norm on $X$ which is equivalent to $\|\cdot\|_{X}$. This implies that the space $\left(X,\|\cdot\|_{r}\right)$ is admissible. Moreover, for $t \in \mathbb{R}$ and $f \in X$

$$
\left\|T_{t} f\right\|_{r}=r\left\|T_{t} f\right\|_{X}+N(f) \leq C_{X}\left(r\|f\|_{X}+N(f)\right) .
$$

It follows that $\left\|T_{t}\right\|_{r} \leq C_{X}$ and thus by Theorem 1

$$
r\left\|\alpha g_{\mathbb{R}}+g_{\mathbb{R}}^{\prime}\right\|+N\left(\alpha g_{\mathbb{R}}+g_{\mathbb{R}}^{\prime}\right) \leq C_{X}\left(\alpha^{2}+\tau^{2}\right)^{1 / 2}\left(r\left\|g_{\mathbb{R}}\right\|_{X}+N\left(g_{\mathbb{R}}\right)\right)
$$

for all $\alpha \in \mathbb{R}$ and all $r>0$. By letting $r \rightarrow 0$ we obtain the desired result. 


\section{THE COROLLARIES}

We observe that the restriction to the real axis of an entire function of exponential type zero is automatically subexponential. So, as an immediate consequence of Theorem 1 we have the following.

Corollary 1. Let $g$ be an entire function of exponential type zero. If the restriction of $g$ to the real axis belongs to some admissible space, then $g$ is constant.

In what follows we will first construct more admissible spaces and then derive the corresponding consequences of Theorem 1 and Theorem 2. Recall from [L-T, p.120] that a function $M(\cdot)$ on $[0, \infty)$ is called an Orlicz function if $M(\cdot)$ is unbounded, continuous, nondecreasing and convex, and satisfies $M(0)=0$. The Orlicz space $L_{M}(\mathbb{R})$ is the space of all measurable functions $f$ on $\mathbb{R}$ such that

$$
\int_{-\infty}^{\infty} M(|f(t)| / \rho) d t<\infty
$$

for some $\rho>0$. The norm in $L_{M}(\mathbb{R})$ is defined by

$$
\|f\|_{M}:=\inf \left\{\rho>0: \int_{-\infty}^{\infty} M(|f(t)| / \rho) d t \leq 1\right\} .
$$

An Orlicz function $M(\cdot)$ is said to satisfy the $\Delta_{2}$-condition both at 0 and at $\infty$ if

$$
\limsup _{t \rightarrow 0} M(2 t) / M(t)<\infty \quad \text { and } \quad \limsup _{t \rightarrow \infty} M(2 t) / M(t)<\infty .
$$

Equivalently, $M(\cdot)$ satisfies the $\Delta_{2}$-condition both at 0 and at $\infty$ if and only if there exists a constant $K>0$ such that

$$
M(2 t) \leq K M(t) \quad \text { for all } \quad t \geq 0 .
$$

Let $M(\cdot)$ be an Orlicz function satisfying $(* * *)$. Then, it is easily verified that the linear span of all simple functions is dense in $L_{M}(\mathbb{R})$. Consequently,

$$
\int_{-\infty}^{\infty} M(|f(t)| / \rho) d t<\infty \quad \text { for all } f \in L_{M}(\mathbb{R}) \text { and } \rho>0 .
$$

Using this observation one can further verify that $L_{M}(\mathbb{R})$ is an admissible space with constant $C_{L_{M}(\mathbb{R})}=1$. Let $T>0$ be such that $M(T)>1$. Consider $f \in L_{M}(\mathbb{R})$. Let

$$
C:=\int_{-\infty}^{\infty} M(|f(t)|) d t<\infty .
$$

Then, the measure of the set $\{t:|f(t)| \geq T\}$ is bounded by $C$. So, every function in $L_{M}(\mathbb{R})$ is subexponential.

Assume now $f$ to be an entire function of exponential type $\tau$ such that its restriction in $\mathbb{R}$ belongs to $L_{M}(\mathbb{R})$, where $M(\cdot)$ is an Orlicz function satisfying $(* * *)$. Let

$$
a:=\int_{-\infty}^{\infty} M(|f(t)|) d t<\infty
$$

We may assume $a>0$. Consider

$$
N(t):=M(t) / a, t \geq 0 .
$$


Then, $N(\cdot)$ preserves the properties of $M(\cdot)$. Moreover, since the set of zeros of $f$ in the real axis is discrete and $M(\cdot)$ is unbounded and nondecreasing, we have

$$
f \in L_{N}(\mathbb{R}) \quad \text { and } \quad\|f\|_{N}=1 .
$$

Theorem 1 applies to $f$ and yields

$$
\left\|\alpha f_{\mathbb{R}}+f_{\mathbb{R}}^{\prime}\right\|_{N} \leq\left(\alpha^{2}+\tau^{2}\right)^{1 / 2}\left\|f_{\mathbb{R}}\right\|_{N}=\left(\alpha^{2}+\tau^{2}\right)^{1 / 2}, \alpha \in \mathbb{R} .
$$

Equivalently, for all $\alpha \in \mathbb{R}$

$$
\int_{-\infty}^{\infty} M\left(\left|\alpha f(t)+f^{\prime}(t)\right| / \sqrt{\alpha^{2}+\tau^{2}}\right) d t \leq a=\int_{-\infty}^{\infty} M(|f(t)|) d t .
$$

This inequality can be extended by approximation to any nondecreasing convex function $M(\cdot)$. Therefore, we have proved the following result which covers Theorem 11.3.3 in [Bo, p.211] obtained by the Complex Analysis method.

Corollary 2. If $f$ is an entire function of exponential type $\tau, M(\cdot)$ a nondecreasing convex function on $[0, \infty)$, then

$$
\int_{-\infty}^{\infty} M\left(\left|\alpha f(t)+f^{\prime}(t)\right| / \sqrt{\alpha^{2}+\tau^{2}}\right) d t \leq \int_{-\infty}^{\infty} M(|f(t)|) d t
$$

for all $\alpha \in \mathbb{R}$.

To find applications of Theorem 2, let $g$ be an entire function of exponential type $\tau$ whose restriction to the real axis is bounded. By the above cited Bernstein theorem we see that the derivative $g_{\mathbb{R}}^{\prime}$ is also bounded. This implies that the restriction $g_{\mathbb{R}}$ is bounded and uniformly continuous, i.e., $g_{\mathbb{R}}$ belongs to the admissible space $B U C(\mathbb{R})$. Let $N(\cdot)$ be any translation-invariant continuous semi-norm on $B U C(\mathbb{R})$. Note that $C_{B U C(\mathbb{R})}=1$. Then, Theorem 2 applies and yields that

$$
N\left(\alpha g_{\mathbb{R}}+g_{\mathbb{R}}^{\prime}\right) \leq\left(\alpha^{2}+\tau^{2}\right)^{1 / 2} N\left(g_{\mathbb{R}}\right) \quad \text { for all } \quad \alpha \in \mathbb{R} .
$$

From this result one can derive many interesting inequalities, which have not been obtained by the Complex Analysis method. For the first example we take $N_{0}(\cdot)$ to be the semi-norm given by

$$
N_{0}(f):=\limsup _{t \rightarrow+\infty}|f(t)|, f \in B U C(\mathbb{R}) .
$$

The above inequality corresponding to $N_{0}(\cdot)$ reads as follows:

$$
\limsup _{t \rightarrow+\infty}\left|\alpha g(t)+g^{\prime}(t)\right| \leq\left(\alpha^{2}+\tau^{2}\right)^{1 / 2} \limsup _{t \rightarrow+\infty}|g(t)|, \alpha \in \mathbb{R} .
$$

This variant of Duffin-Schaeffer's inequality gives the relation between the asymptotic behavior at infinity of functions and their derivatives. Such a result seems to be new.

For the second example we take $M(\cdot)$ to be an Orlicz function on $[0, \infty)$ with $M(0)=0$. Define

$$
\omega_{M}(f):=\limsup _{T \rightarrow+\infty} \frac{1}{2 T} \int_{-T}^{T} M(|f(t)|) d t, f \in B U C(\mathbb{R}),
$$

and

$$
N_{M}(f):=\inf \left\{\rho>0: \omega_{M}(f / \rho) \leq 1\right\}, f \in B U C(\mathbb{R}) .
$$


It is easily verified that $N_{M}(\cdot)$ is a translation-invariant semi-norm. Let $D>0$ be such that $M\left(D^{-1}\right) \leq 1$. Then, for $f \in B U C(\mathbb{R})$ we have

$$
\begin{gathered}
M\left(|f(t)| /\left(D \cdot\|f\|_{\infty}\right)\right) \leq M\left(D^{-1}\right) \leq 1, \\
\omega_{M}\left(f /\left(D \cdot\|f\|_{\infty}\right)\right) \leq 1 \quad \text { and } \quad N_{M}(f) \leq D\|f\|_{\infty} .
\end{gathered}
$$

Theorem 2 is applicable to $N_{M}(\cdot)$ with the above function $g$ and yields that

$$
N_{M}\left(\alpha g_{\mathbb{R}}+g_{\mathbb{R}}^{\prime}\right) \leq\left(\alpha^{2}+\tau^{2}\right)^{1 / 2} N_{M}\left(g_{\mathbb{R}}\right) \quad \text { for all } \quad \alpha \in \mathbb{R}
$$

By a same procedure as in the proof of Corollary 2 we find from above that

$$
\omega_{M}\left(\left(\alpha g_{\mathbb{R}}+g_{\mathbb{R}}^{\prime}\right) / \sqrt{\alpha^{2}+\tau^{2}}\right) \leq \omega_{M}\left(g_{\mathbb{R}}\right) \quad \text { for all } \quad \alpha \in \mathbb{R} .
$$

Furthermore, it can be extended through approximation to any nondecreasing convex function $M(\cdot)$. By taking $\alpha=0$ we have

$$
\omega_{M}\left(g_{\mathbb{R}}^{\prime} / \tau\right) \leq \omega_{M}\left(g_{\mathbb{R}}\right)
$$

This inequality corresponds to Theorem 11.3.3 in [Bo, p.211]. For the moment we collect these results as follows.

Corollary 3. Let $g$ be an entire function of exponential type $\tau$, which is bounded in the real axis. Then, for all $\alpha \in \mathbb{R}$,

$$
\limsup _{t \rightarrow+\infty}\left|\alpha g(t)+g^{\prime}(t)\right| \leq\left(\alpha^{2}+\tau^{2}\right)^{1 / 2} \limsup _{t \rightarrow+\infty}|g(t)| .
$$

Moreover, for any nondecreasing convex function $M(\cdot)$ on $[0, \infty)$ and any $\alpha \in \mathbb{R}$,

$$
\limsup _{T \rightarrow+\infty} \frac{1}{2 T} \int_{-T}^{T} M\left(\frac{\left|\alpha g(t)+g^{\prime}(t)\right|}{\sqrt{\alpha^{2}+\tau^{2}}}\right) d t \leq \limsup _{T \rightarrow+\infty} \frac{1}{2 T} \int_{-T}^{T} M(|g(t)|) d t .
$$

As another example we consider applications in the context of almost periodic functions. Recall that a continuous function $h$ on $\mathbf{R}$ is called almost periodic (a.p.) if the set $\left\{h^{t}: t \in \mathbb{R}\right\}$ of all all translates of $h$ is relatively compact in $C(\mathbb{R})$ (see [Ka, pp. 155-169]). Denote by $A P(\mathbb{R})$ the Banach space of all a.p. functions on $\mathbb{R}$ with the supremum norm. It is known that an a.p. function is uniformly continuous. Therefore, $A P(\mathbb{R})$ is a translation-invariant subspace of the admissible space $B U C(\mathbb{R})$ and hence it is also admissible. In the previous section we have defined the local spectrum $\operatorname{Sp}_{\mathcal{T}}(h)$ for each $h \in A P(\mathbb{R})$. There is another spectral notation for a.p. functions, called the Bohr spectrum, which is defined as follows. Note that for each a.p. function $h$ the limit

$$
M(h):=\lim _{T \rightarrow+\infty} \frac{1}{2 T} \int_{-T}^{T} h(t) d t
$$

exists. The Bohr spectrum $\Lambda_{B}(h)$ of $h$ is defined as the set of all $\xi \in \mathbb{R}$ such that $M\left(h(t) e^{-i \xi t}\right) \neq 0$. This is a countable set. It is shown in [Hu, Proposition 4.3.10] that the closure of $\Lambda_{B}(h)$ is equal to the local spectrum $\operatorname{Sp}_{\mathcal{T}}(h)$.

Assume that $h$ is a.p. and its Bohr spectrum is contained in the finite interval $[-\tau, \tau]$. Then, $h \in A P(\mathbb{R})^{\mathcal{T}}([-\tau, \tau])$. As seen in Lemma 1 , the operator $B$ obtained by restricting the derivative to the spectral subspace $A P(\mathbb{R})^{\mathcal{T}}([-\tau, \tau])$ is a bounded operator with norm $\|B\| \leq \tau$. We have

$$
h^{t}=T_{t} h=e^{t B} h, t \in \mathbb{R} .
$$


Consider the function

$$
H(z):=\left(e^{z B} h\right)(0), z \in \mathbf{C} .
$$

Then, $H(z)$ is an entire function and $h(t)=H(t)$ for all $t \in \mathbb{R}$. Note that

$$
|H(z)| \leq\left\|e^{z B} h\right\|_{\infty} \leq e^{\|z B\|} \cdot\|h\|_{\infty} \leq e^{\tau|z|} \cdot\|h\|_{\infty} .
$$

We find that $H(z)$ is an entire function of exponential type not exceeding $\tau$. By applying Corollary 3 to $H(z)$ we obtain the following result.

Corollary 4. Let $h \in A P(\mathbb{R})$ be such that its Bohr spectrum is contained in the finite interval $[-\tau, \tau]$. Then, $h$ can be extended to an entire function of exponential type not exceeding $\tau$. Moreover, for any nondecreasing convex function $M(\cdot)$ on $[0, \infty)$ and any $\alpha \in \mathbb{R}$,

$$
\limsup _{T \rightarrow+\infty} \frac{1}{2 T} \int_{-T}^{T} M\left(\frac{\left|\alpha h(t)+h^{\prime}(t)\right|}{\sqrt{\alpha^{2}+\tau^{2}}}\right) d t \leq \limsup _{T \rightarrow+\infty} \frac{1}{2 T} \int_{-T}^{T} M(|h(t)|) d t .
$$

The subsequent construction of admissible spaces through Peetre's interpolation method [Pee] (see also [L-T, pp. 216-232] and [B-L]) gives many Bernstein type inequalities which have not been derived by the Complex Analysis method in [Bo]. To begin, let $X_{1}, X_{2}$ be admissible spaces. The space $X_{1}+X_{2}$ is normed by

$$
\|z\|_{X_{1}+X_{2}}:=\inf \left\{\left\|x_{1}\right\|_{X_{1}}+\left\|x_{2}\right\|_{X_{2}}: z=x_{1}+x_{2}\right\} .
$$

The space $X_{1} \cap X_{2}$ is normed by

$$
\|z\|_{X_{1} \cap X_{2}}:=\max \left\{\|z\|_{X_{1}},\|z\|_{X_{2}}\right\} \text {. }
$$

It is easily verified that both of the spaces $X_{1}+X_{2}$ and $X_{1} \cap X_{2}$ are admissible Banach spaces. In general, for every $a>0, b>0$ let $k(\cdot, a, b)$ be the equivalent norm on $X_{1}+X_{2}$ defined by

$$
k(z, a, b):=\inf \left\{a\left\|x_{1}\right\|_{X_{1}}+b\left\|x_{2}\right\|_{X_{2}}: z=x_{1}+x_{2}\right\} .
$$

We have

$$
C_{\left(X_{1}+X_{2}, k(\cdot, a, b)\right)} \leq \max \left(C_{X_{1}}, C_{X_{2}}\right) .
$$

Let, in addition, $Y$ be a Banach space with a normalized unconditional basis $\left\{y_{n}\right\}_{n=1}^{\infty}$ whose unconditional constant is one. Let $\mathbf{a}:=\left\{a_{n}\right\}_{n=1}^{\infty}$ and $\mathbf{b}:=\left\{b_{n}\right\}_{n=1}^{\infty}$ be sequences of positive numbers such that $\sum_{n=1}^{\infty} \min \left(a_{n}, b_{n}\right)<\infty$. The space $K\left(X_{1}, X_{2}, Y, \mathbf{a}, \mathbf{b}\right)$, resp. $\tilde{K}\left(X_{1}, X_{2}, Y, \mathbf{a}, \mathbf{b}\right)$, is defined to be the space of all functions $z \in X_{1}+X_{2}$ such that $\sum_{n=1}^{\infty} k\left(z, a_{n}, b_{n}\right) y_{n}$ converges, resp. $\left\{\sum_{n=1}^{m} k\left(z, a_{n}, b_{n}\right) y_{n}: m \in \mathbf{N}\right\}$ is bounded, and normed by

$$
\|z\|_{K\left(X_{1}, X_{2}\right)}=\|z\|_{\tilde{K}\left(X_{1}, X_{2}\right)}:=\sup _{m}\left\|\sum_{n=1}^{m} k\left(z, a_{n}, b_{n}\right) y_{n}\right\|_{Y} .
$$

They satisfy

$$
X_{1} \cap X_{2} \subset K\left(X_{1}, X_{2}, Y, \mathbf{a}, \mathbf{b}\right) \subset \tilde{K}\left(X_{1}, X_{2}, Y, \mathbf{a}, \mathbf{b}\right) \subset X_{1}+X_{2} .
$$

Moreover, since $X_{1}+X_{2}$ is admissible one can prove that $K\left(X_{1}, X_{2}, Y, \mathbf{a}, \mathbf{b}\right)$ is also admissible and

$$
C_{K\left(X_{1}, X_{2}\right)} \leq \max \left(C_{X_{1}}, C_{X_{2}}\right) .
$$


Corollary 5. Let $\tilde{K}\left(X_{1}, X_{2}, Y, \mathbf{a}, \mathbf{b}\right)$ be an interpolation space given as above. If $g$ is an entire function of exponential type $\tau$ such that its restriction to the real axis belongs to $\tilde{K}\left(X_{1}, X_{2}, Y, \mathbf{a}, \mathbf{b}\right)$, then its derivative is also in $\tilde{K}\left(X_{1}, X_{2}, Y, \mathbf{a}, \mathbf{b}\right)$ and, for all $\alpha \in \mathbb{R}$,

$$
\left\|\alpha g_{\mathbb{R}}+g_{\mathbb{R}}^{\prime}\right\|_{K\left(X_{1}, X_{2}\right)} \leq\left(\alpha^{2}+\tau^{2}\right)^{1 / 2} \cdot \max \left(C_{X_{1}}, C_{X_{2}}\right) \cdot\left\|g_{\mathbb{R}}\right\|_{K\left(X_{1}, X_{2}\right)} .
$$

Moreover, if the restriction $g_{\mathbb{R}}$ is in the admissible subspace $K\left(X_{1}, X_{2}, Y, \mathbf{a}, \mathbf{b}\right)$, then so is the derivative $g_{\mathbb{R}}^{\prime}$.

Proof. We only need to check the estimate of the norm. Since the space $X_{1}+X_{2}$ is admissible, we find by Theorem 1 that both of the restrictions in the real axis $g_{\mathbb{R}}$ and $g_{\mathbb{R}}^{\prime}$ are in $X_{1}+X_{2}$. Let $n \in \mathbb{N}$. Then,

$$
C_{\left(X_{1}+X_{2}, k\left(\cdot, a_{n}, b_{n}\right)\right)} \leq \max \left(C_{X_{1}}, C_{X_{2}}\right) .
$$

Fix $\alpha \in \mathbb{R}$. By Theorem 1 again,

$$
k\left(\alpha g_{\mathbb{R}}+g_{\mathbb{R}}^{\prime}, a_{n}, b_{n}\right) \leq\left(\alpha^{2}+\tau^{2}\right)^{1 / 2} \cdot \max \left(C_{X_{1}}, C_{X_{2}}\right) \cdot k\left(g_{\mathbb{R}}, a_{n}, b_{n}\right) .
$$

Since the basis $\left\{y_{n}\right\}_{n=1}^{\infty}$ is unconditional with constant one, the above implies that

$$
\left\|\alpha g_{\mathbb{R}}+g_{\mathbb{R}}^{\prime}\right\|_{K\left(X_{1}, X_{2}\right)} \leq\left(\alpha^{2}+\tau^{2}\right)^{1 / 2} \cdot \max \left(C_{X_{1}}, C_{X_{2}}\right) \cdot\left\|g_{\mathbb{R}}\right\|_{K\left(X_{1}, X_{2}\right)},
$$

proving the result.

A special case of Peetre's interpolation spaces is Lions-Peetre's interpolation spaces $\left[X_{1}, X_{2}\right]_{\theta, p}$ for $0<\theta<1$ and $1 \leq p \leq \infty$, see [L-P]. It is shown in $[\mathrm{L}-\mathrm{P}]$ (see also $\left[\mathrm{L}-\mathrm{T}\right.$, Theorem 2.g.18, p.228]) that the space $\left[L_{p_{1}}(\mathbb{R}), L_{p_{2}}(\mathbb{R})\right]_{\theta, q}$ with $1 \leq p_{1}<p_{2}<\infty, 0<\theta<1,1 \leq q \leq \infty$ is equal, up to an equivalent norm, to the Lorentz space $L_{p, q}(\mathbb{R})$ (see [L-T, p.142] for the definition), where $1 / p=(1-\theta) / p_{1}+\theta / p_{2}$. Thus, our Corollaries 1 and 4 apply to the spaces $L_{p, q}(\mathbb{R})$, where $1<p<\infty, 1 \leq q<\infty$. Now, a consequence of Corollary 1 reads as follows: If $g$ is an entire function of exponential type zero such that its restriction to the real axis belongs to $L_{p, q}(\mathbb{R})$ for some $1<p<\infty$ and $1 \leq q<\infty$, then $g$ is a constant. Such results involving the estimate of the distribution function of an entire function seem to be new.

\section{Comments And Discussions}

We first remark that Theorems 1 and 2 and their consequences can be extended to vector-valued entire functions taking values in a Banach space $X$. The admissibility of a translation-invariant subspace $E(X)$ of the locally Bochner integrable space $L_{l o c}^{1}(\mathbb{R}, X)$ can be defined similarly as in Definition 1 . We note that the Banach function spaces $E(X)$ which are admissible in the sense of $[\mathrm{R}-\mathrm{S}]$ are also admissible in our sense.

One important tool used by Boas and Levin in their books to derive the Bernstein type inequalities is the Paley-Wiener theorem, which can be proved by the Plancherel theorem (see [Bo, pp. 105-106]). We would like to make the reader aware that in our proof the Plancherel theorem is also used in an implicit way. Namely, the regularity of the group algebra $L^{1}(\mathbb{R})$ is involved in the spectral theory of Arveson and for the proof of the regularity of $L^{1}(\mathbb{R})$ one should use the Plancherel theorem, see [Lo, Corollary, p.146]. Our method of deriving Bernstein type inequalities is different from the Complex Analysis method of Boas [Bo] and Levin [Le], and the results are more general. In a subsequent paper, using the same 
idea, we will derive Bernstein type inequalities for entire functions of exponential type whose restriction to the real axis belongs to so-called rearrangement-invariant spaces defined on $\mathbb{R}$. On such a space the translation group is generally not strongly continuous, but we can use the spectral theory that has been recently established in $[\mathrm{Hu}]$.

\section{ACKNOWLEDGEMENT}

The author thanks Prof. R. Nagel and Dr. J. van Neerven for their valuable discussions.

\section{REFERENCES}

[Ar] W. ARveson: On groups of automorphisms of operator algebras. J. Funct. Anal. 15 (1974), 217-243. MR 50:1016

[B-C] F. F. Bonsall and M. J. CrabB: The spectral radius of a Hermitian element of a Banach algebra. Bull. London Math. Soc. 2 (1970), 178-180. MR 42:853

[Be] S. Bernstein: Sur une les propriété des fonctions entiéres. C. R. Acad. Sci. Paris 176 (1923), 1603-1605.

[B-L] J. Bergh AND J. LÖFström: Interpolation Spaces, an Introduction. Springer-Verlag 1976. MR 58:2349

[Bo] R. H. BoAs: Entire Functions. Academic Press Inc., Publishers, New York 1954. MR 16:914f

[Do] H. R. Dowson: Spectral Theory of Linear Operators. Academic Press, London, New York, San Francisco 1978. MR 80c: 47022

[D-S] R. J. Duffin And A. C. Schaeffer: Some inequalities concerning functions of exponential type. Bull. Amer. Math. Soc. 43 (1937), 554-556.

[Ev] D. E. Evans: On the spectrum of a one-parameter strongly continuous representation. Math. Scand. 39 (1976), 80-82. MR 55:3873

[Hu] S.-Z. HuAng: Spectral Theory for Non-Quasianalytic Representations of Locally Compact Abelian Groups. monograph 1995.

[Jo] P. E. T. Jorgensen: Spectral theory for one-parameter groups of isometries. J. Math. Anal. Appl. 168 (1992), 131-146. MR 93e:47048

[Ka] Y. Katznelson: An Introduction to Harmonic Analysis. John Wiley \& Sons, Ney York 1968. MR 40:1734

[K-S-T] E. Kochneff, Y. Sagher and R. Tan: On Bernstein inequality. Illinois J. Math. 36 (1992), 297-309. MR 93h:42008

[Le] B. JA. Levin: Distributions of Zeros of Entire Functions. Translations of Mathematical Monographs, Vol. 5, Amer. Math. Soc., Providence (R.I.), 1964.

[Lo] L. H. Loomis: An Introduction to Abstract Harmonic Analysis. D. Van Nostrand Comp., Princeton (N.J.) 1953. MR 14:883c

[Lor] G. G. LoRentz: Approximation of Functions. Holt, Rinehart and Winston, New York 1966. MR 35:4642

[L-P] J. L. Lions And J. Peetre: Sur une classe d'espaces d'interpolation. Publ. Math. Inst. Hautes Etudes Sci. 19 (1964), 5-68. MR 29:2627

[L-T] J. Lindenstrauss and L. Tzafriri: Classical Banach Spaces II, Function Spaces. Springer-Verlag 1979. MR 81c:46001

[N-H] R. NAgel And S.-Z. Huang: Spectral mapping theorem for $C_{0}$-groups satisfying nonquasianalytic growth conditions. Math. Nachr. 169 (1994), 207-218. MR 95h:47055

[Ol] D. Olesen: On norm continuity and compactness of spectrum. Math. Scand. 35 (1974), 223-236. MR 51:10532

[Pal] T. W. PALmer: Banach Algebras and the General Theory of *-Algebras I: Algebras and Banach Algebras. Cambridge Univ. Press, London 1994. MR 95c:46002

[Paz] A. PAZY: Semigroups of Linear Operators and its Applications to Partial Differential Equations. Springer-Verlag 1983. MR 85g:47061

[Ped] G. K. Pedersen: $C^{*}-$ Algebras and their Automorphism Groups. Academic Press, London 1979. MR 81e:46037 
[Pee] J. PeEtre: A theory of interpolation of normed spaces. Notas de mathematica Brazil 39 (1968), 1-86. MR 39:4662

[R-S] F. RÄBiger and R. Schnaubelt: The spectral mapping theorem for evolution semigroups on space of vector-valued functions. Semigroup Forum 52 (1996), 225-239. CMP 96:07

[Re] R. M. REDheffer: Completeness of sets of complex exponentials. Adv. In Math. 24 (1977), 1-62. MR 56:5852

[Si] A. M. Sinclair: The norm of a hermitian element in a Banach algebra. Proc. Amer. Math. Soc. 28 (1971), 446-450. MR 43:921

[Ti] A. F. Timan: Theory of Approximation of Functions of a Real Variable. International Series of Monographs on Pure and Applied Mathematics, Vol. 34, Pergamon Press, 1963. MR 33:465

Mathematisches Institut, Universität Tübingen, Auf der Morgenstelle 10, D-72076 Tübingen, F. R. Germany

E-mail address: huse@michelangelo.mathematik.uni-tuebinegn.de 